\title{
Clip reconstruction of a large right MCA bifurcation aneurysm. Case report
}

\author{
A. Giovani ${ }^{1}$, Angela Neacsu, Ana Gheorghiu, R.M. Gorgan \\ Department of Neurosurgery \\ Clinical Hospital Bagdasar-Arseni, Bucharest \\ ${ }^{1}$ Student
}

\begin{abstract}
We report a case of complex large middle cerebral artery (MCA) bifurcation aneurysm that ruptured during dissection from the very adherent MCA branches but was successfully clipped and the MCA bifurcation reconstructed using 4 Yasargill clips. Through a right pterional craniotomy the sylvian fissure was largely opened as to allow enough workspace for clipping the aneurysm and placing a temporary clip on M1. The pacient recovered very well after surgery and was discharged after 1 week with no neurological deficit. Complex MCA bifurcation aneurysms can be safely reconstructed using regular clips, without the need of using fenestrated clips or complex by-pass procedures.
\end{abstract}

Key words: MCA bifurcation; Yasargil clips; clip reconstruction.

\section{Introduction}

The MCA aneurysms represent $20-25 \%$ of all intracranial aneurysms. Given their complex anatomy including broad necks, trifurcated anatomy and the high risk to occlude a small branch whose origin cannot be seen on angiography when detaching the coils these aneurysm are considered unfavorable for endovascular treatment. Because they are easy to reach in the Sylvian fissure and in that region the fissure is large enough to allow complex reconstruction and bypass techniques if necessary the best treatment for these lesions is surgical clipping. Several studies showed the surgical results to be superior to endovascular results. $(3,4)$ This article reports the reconstruction of MCA branches by applying several parallel clips to a large MCA aneurysm that could not be dissected and ruptured while being dissected from one of the branches. $(6,7,12)$

\section{Case report}

A 59 years old right handed woman with multiple associated pathology including basocellular nose carcinoma operated 2 years prior, glaucoma, diabetes mellitus, and psychiatric symptoms including depression and bipolar disorder, presented for intense headache worsened over the last two weeks. The patient had a two years history o intermittent headache. A CT angiography 1 week prior admission indicated the presence 
of a right MCA bifurcation aneurysm. The lesion was confirmed on the angiography but the neck was very difficult to describe because of 4 branches of the MCA surrounding the aneurism given the very close. This aspect was probably due to the bifurcation of each of the 2 M2's immediately after emergence from M1. No subarachnoid hemorrhage was visible on CT so it was an incidental discovery. The rotational angio $\mathrm{CT}$ in our department was more useful than the angiography because it showed the relation of the aneurysm with the MCA branches whereas on angiography the branches superimposed on the contour of the aneurysm and the neck could not be distinguished.

The patient was positioned supine with the head rotated $45^{\circ}$ to the left. A right frontotemporal standard craniotomy was performed. After the dural incision the Sylvian fissure was opened from distal to proximal. The M1 was identified with a large bilobulated aneurysm at its bifurcation. Immediately after origin from M1 both M2 bifurcated and all 4 branches engulfing the aneurysm prevented the operator to gain access to the neck. A very adherent branch separated the two lobes of the aneurysm. When attempting to dissect the dome from this branch, given the very thin wall at this level, a punctiforme hole was created in the aneurysm but the wall did not tear. A temporary clip was applied on M1 and removed 1 minute later after a definitive curved clip was applied on the hole in the aneurysm and the bleeding stopped.

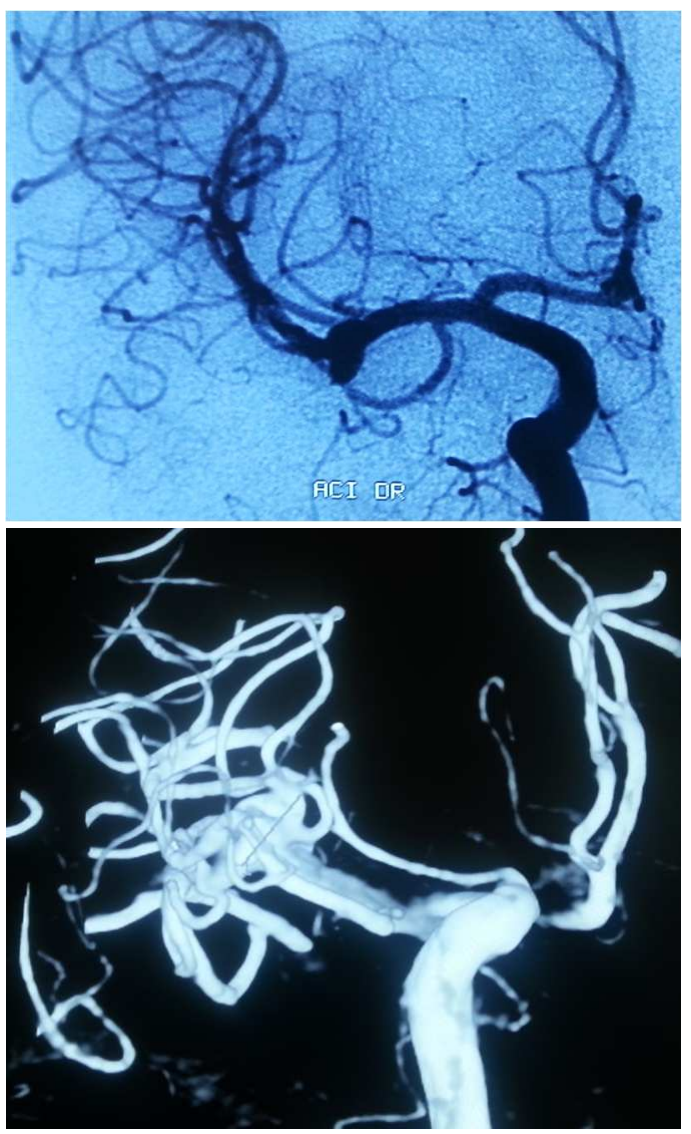

Figure 1 - Preop. Angiograpgy and angio-CT

The aneurysm was next reconstructed using two more curved clips on the bigger (frontal) lobule with care to spare the frontal M2 branch adherent to the dome. Than the temporal lobule was reconstructed with a 4th clip applied between two temporal branches. (Figures 1 and 2). No retractor was needed during surgery, the suction tube was used as a retractor (1). Also the importance of Sylvian fissure opening should be stated. Before closure the arterial pressure was raised to make sure there was no bleeding, and the clipping was controlled with a Doppler probe to make 
DOI: 10.2478/romneu-2014-0017

sure no MCA branch is occluded and no aneurism remnant was left. The dura was closed and circullary suspended to the fascia, the bone flap fixed in place with separated sutures and the scalp closed.

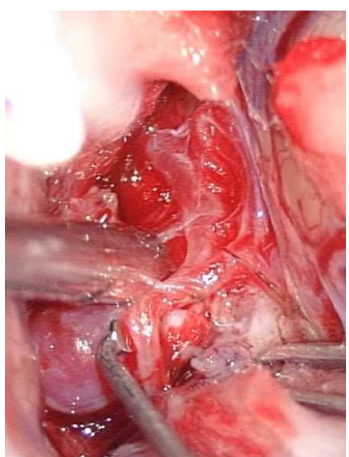

A

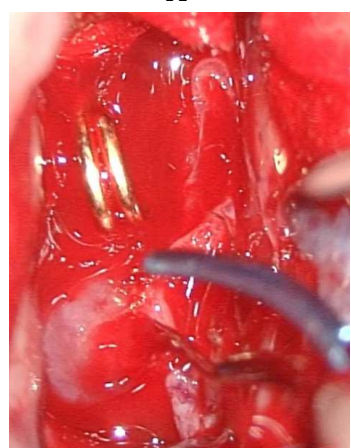

C

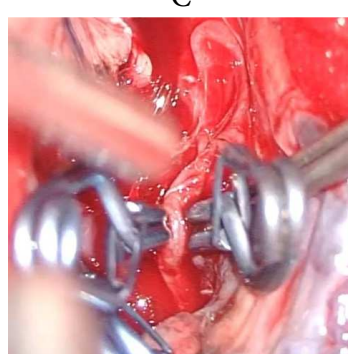

$\mathrm{E}$

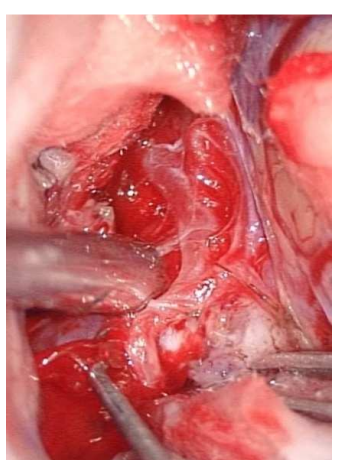

B

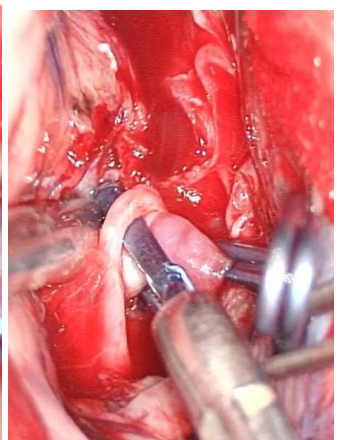

$\mathrm{D}$

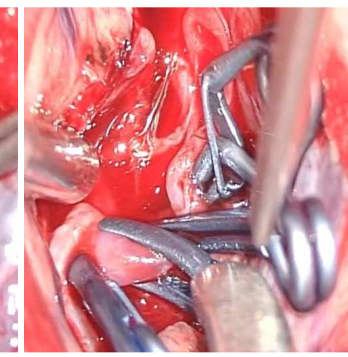

$\mathrm{F}$
Figure 2 - A. Tight adherent branch dissection; B. rupture of the aneurysm; C. first clip including the rupture site; D. second clip inserted under a frontal branch; E. third clip on the small temporal aneurysm lobule; F. fourth curved clip to include the rest of the aneurysm
The patient showed no neurological deficit after the surgery and she was discharged 7 days after surgery. The postoperative angiography showed a good flow in the right MCA peripheral branches and the disappearance of the aneurysm.
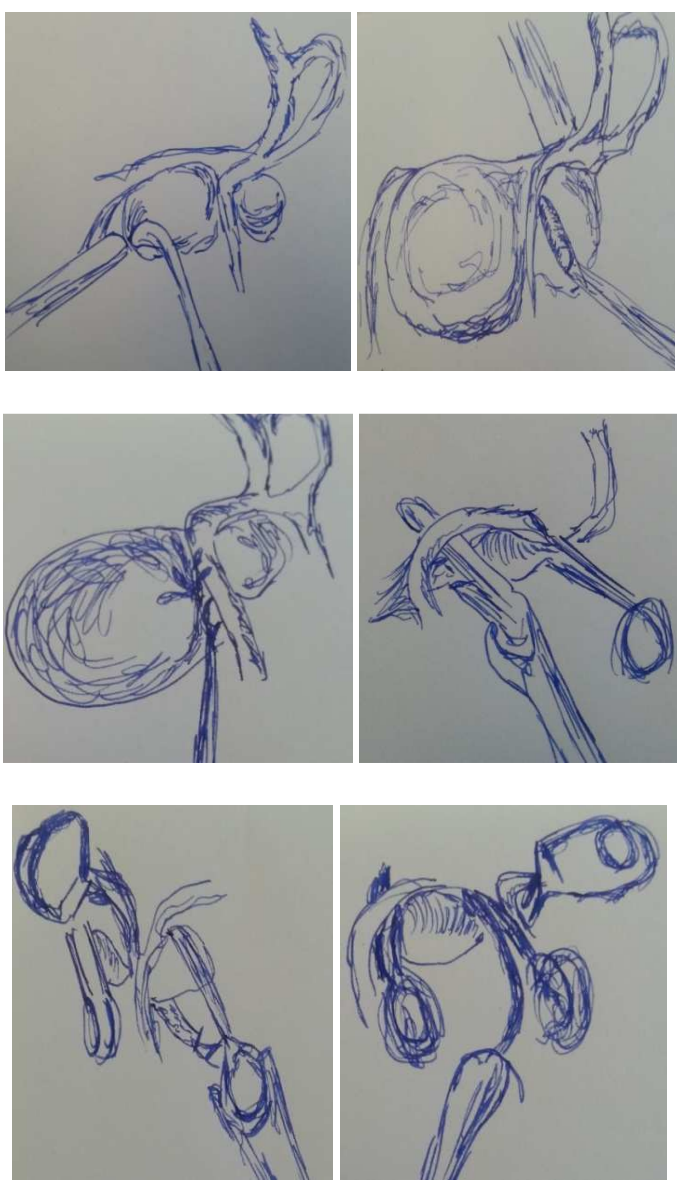

Figure 3 - Graphic depiction of the dissection and clipping starting with freeing the dome from the highly adherent branches on its surface followed by rupture and clip reconstruction 

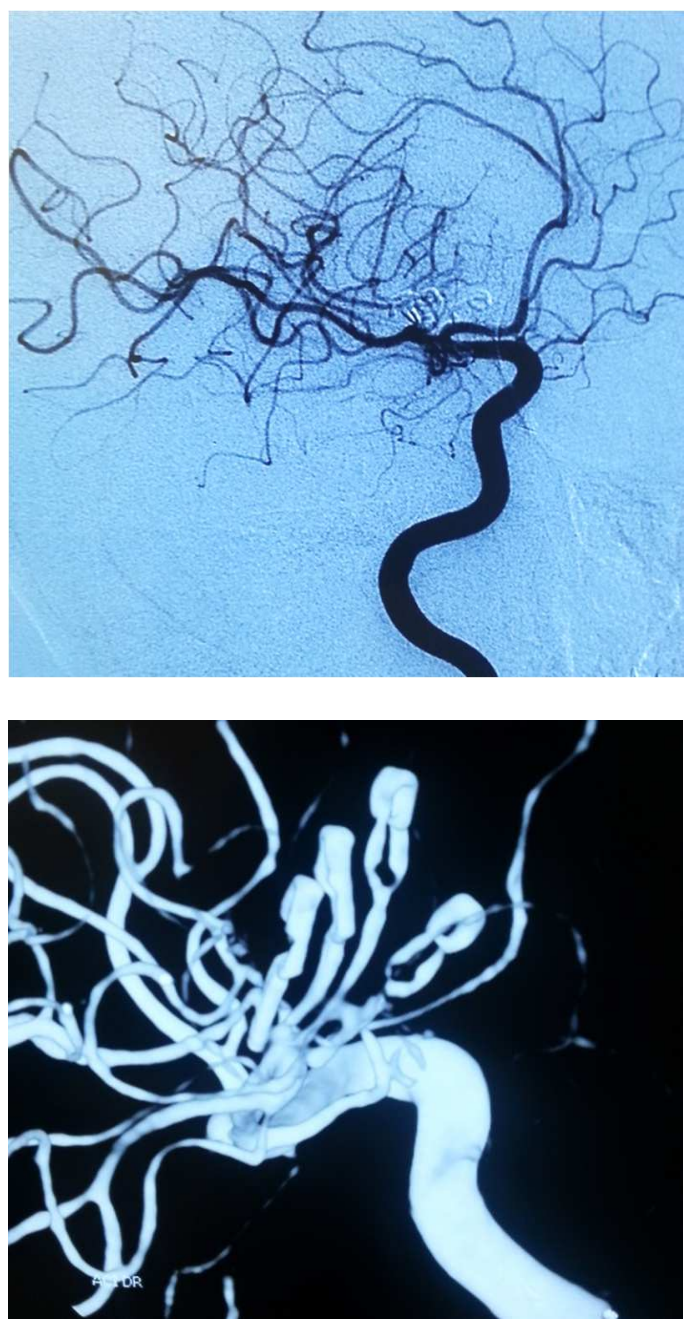

Figure 4 - Postoperative angiography and Angio-CT

\section{Discussion}

Treatment of complex MCA bifurcation aneurysms represents a challenge both for the endovascular surgeon given the broad neck and for the neurosurgeon given that major branches of MCA can be adherent to the aneurism wall. The purpose of surgery is exclusion of the aneurysm from circulation, with preservation of normal vascular anatomy while minimizing the risk of recurrence at the clipping site. $(9,10,11)$ The latter is achieved by appropriate placement of the clips used to reconstruct the aneurysm without letting any dog ears, and this can be difficult to achieve in cases where the clip slides on atheromatous vessels. It is very important to control the clipping in order to make sure that no branch is occluded and there is no aneurysm remnant. Even though it is not available in our service indocyanine-green is very usefull as a proof that the perfusion of the branches distal to the aneurysm is adequate.

We reconstructed the aneurysm using 4 Yasargil clips two straight and two curved applied in such a fashion as to clip the base of the aneurysm without injuring or obliterating the branches that crossed on the surface of the aneurysm. $(4,5,8)$

In cases where the branches cannot be dissected from the fragile aneurysm wall, using fenestrated clips alone or stacked together to create tubes around the branch should be considered. (13) Unfortunately we had no such clips available for this surgery.

In gigant aneurysms or in those large unclipable, more difficult repair techniques including excision of the aneurysm and reanastomosing the vessels, or simply resuturing the arterial wall after resecting the aneurysm with or without a high flow or a low flow bypass are to be taken into account $(2,5)$. Of note is also the technique of bipolar shrinkage of the aneurysm which makes it smaller, more easily to dissect from the adherences and more clipable. This technique though is very risky in aneurysms with very thin walls and in those very adherent to the 
DOI: 10.2478/romneu-2014-0017

branches. The technique used is a good option for clipping large MCA bifurcation aneurysms whose dome and neck cannot be fully dissected from the adherences.

\section{Correspondence}

Giovani Andrei M.D. PhD Stud.

Department of Neurosurgery

Clinical Hospital Bagdasar-Arseni, Bucharest

e-mail: giovani.andrei@gmail.com

\section{References}

1. Robert F. Spetzler, M.D., and Nader Sanai, M.D. 2012. The quiet revolution: retractorless surgery for complex vascular and skull base lesions. Clinical Article. J Neurosurg 116:291-300.

2. Mrak, Goran; Paladino, Josip; Stambolija, Vasilije; Jacob Nemir; Laligam N. Sekhar. 2014. Treatment of Giant and Large Fusiform MCA Aneurysms With Excision and Interposition Radial Artery Graft in a Four Year Old Child. Neurosurgery., 10:172-177

3. Ulm, Arthur J.; Fautheree, Gregory L.; Tanriover, Necmettin, 2008, Microsurgical and Angiographic Anatomy of Middle Cerebral Artery Aneurysms: Prevalence and Significance of Early Branch Aneurysms; More; Neurosurgery. 62(5):ONS344-ONS353.

4. Rodríguez-Hernández, Ana; Gabarrós, Andreu; Lawton, Michael T.; 2012; Contralateral Clipping of Middle Cerebral Artery Aneurysms: Rationale, Indications, and Surgical Technique Neurosurgery. 71():ons116-ons124.

5. Nossek, Erez; Costantino, Peter; Eisenberg, Mark; More ;2014; Internal Maxillary Artery to Middle Cerebral Artery Bypass: Infratemporal Approach for SubcranialIntracranial (SC-IC) Bypass Neurosurgery., POST ACCEPTANCE, 10 March 2014
6. Michel Bojanowski, M.D., Robert F. Spetzler M.D., and L. Philip Carter, M.D. 1988. Reconstruction of the MCA bifurcation after excision of a giant aneurysm technical note. J Neurosurg 68:974-977,

7. Yuichiro Tanaka, M.D., Shigeaki Kobayashi, M.D., Kazuhlko Kyoshima, M.D., and Kenichiro Sugita, M.D. 1994; Multiple clipping technique for large and giant internal carotid artery aneurysms and complications: angiographic analysis; J Neurosurg 80:635-642.

8. Leena Kivipelto, M.D., Ph.D., Mika Niemelä, M.D., Ph.D., Torstein Meling, M.D., Ph.D., Martin Lehecka, M.D., Ph.D., Hanna Lehto, M.D., and Juha Hernesniemi, M.D., Ph.D. ;Feb 2014; Bypass surgery for complex middle cerebral artery aneurysms: impact of the exact location in the MCA tree: Clinical article Journal of Neurosurgery / Vol. 120 / No. 2, Pages 398-408

9. Daniel L. Surdell, M.D., Ziad A. Hage, M.D., Christopher S. Eddleman, M.D., Ph.D., Dhanesh K. Gupta, M.D., Bernard R. Bendok, M.D., and H. Hunt Batjer, M.D.; Feb 2008 ; Revascularization for complex intracranial aneurysms . Neurosurgical Focus / Vol. 24 / No. 2, Page E21

10.Clatterbuck, Richard E.; Galler, Robert M.; Tamargo, Rafael J ; October 2006; Orthogonal Interlocking Tandem Clipping Technique for the Reconstruction of Complex Middle Cerebral Artery Aneurysms.; Neurosurgery. 59(4):ONS-347-ONS-352,.

11.Rodríguez-Hernández, Ana; Lawton, Michael T.; June 2012; Flash Fluorescence With Indocyanine Green Videoangiography to Identify the Recipient Artery for Bypass With Distal Middle Cerebral Artery Aneurysms: Operative Technique Neurosurgery. 70():ons209-ons220. 12.Heros, Roberto C.; Fritsch, Michael J. Surgical Management of Middle Cerebral Artery Aneurysms Neurosurgery. 48(4):780-786, April 2001.

13.Yang, Isaac M.D., Ph.D.; Lawton Michael T. M.D. 2008; Clipping of Complex Aneurysms With Fenestration Tubes: Application and Assessment of Three Types of Clip Techniques; Neurosurgery: Volume 62 - Issue 5 - p ONS371-ONS379. 